\title{
Dielectric Phase Transition Behaviour Study of Dry Route Derived
} $\left(\mathrm{Ba}_{0.5} \mathrm{Sn}_{0.5}\right) \mathrm{TiO}_{3}$ Ceramics

\author{
Bhojraj Bhandari ${ }^{* *}$, Bhadra Prasad Pokheral ${ }^{2}$ \\ ${ }^{1}$ Department of Physics, Goldengate International College, Kathmandu, Nepal \\ 2 Pulchowk Engineering Campus, Institute of Engineering, Tribhuvan University, Pulchowk, Lalitpur, Nepal \\ *Corresponding E-mail: boydbhandari@gmail.com \\ (Received: Sept. 21, 2019; Revised: Dec. 13, 2019 \& Accepted: Dec. 15, 2019)
}

\begin{abstract}
Ceramic materials display a wide range of properties that facilitate their use in many different product areas. Currently, there has been keen interest in the field of ceramic materials due to their excellent mechanical and physical properties. Barium Stannate Titanate (BST) is a binary solid solution system composed of ferroelectric Barium titanate and non-ferroelectric barium titanate. In this study, the phase transition behavior of $\left(\mathrm{Ba}_{1-\mathrm{x}} \mathrm{Sn}_{\mathrm{x}}\right)$ $\mathrm{TiO}_{3}(\mathrm{x}=0.5)(\mathrm{BST})$ ceramics was obtained by the dry-route method. The previous studies were based on $\mathrm{Sn}^{2+}$ on the Ti site with varying values of $\mathrm{x}$. The powders after calcination are compacted in the form of pellets using a hydraulic press at an optimized load above $70 \mathrm{KN}$. The experimental density of our sample measured by liquid displacement method with glycerin was lower than theoretical density, giving the shape is highly dense with low porosity. The structure shows that on increasing the $\mathrm{Sn}^{2+}$ content volume decreases due to the size of $\mathrm{Sn}^{2+}$, which is smaller than that of $\mathrm{Ba}^{2+}$, in comparison to $\mathrm{BaTiO}_{3}$. As the demand of lead-free environment-friendly sensor is increasing, thus obtained BST has great applications as a sensor material in modern electronic devices.
\end{abstract}

Keywords: Porosity measurement, phase transitions, dielectric property, Curie-Wiess law

\section{Introduction}

The development of Science \& technology unified people all over the world like a single-family. While discussing with research and innovation, scientists as well as researchers have been attracted to those materials which are convenient, economical and more applicable. During the last few decades, people are being attracted to other materials rather than metals, like ceramic, polymer glass etc.

The word ceramics derived from the Greek word keramikos [1]. A ceramic is an inorganic, nonmetallic solid prepared by the action of heat and subsequent cooling. Ceramic materials may have a crystalline or partly crystalline structure, or may be amorphous (e.g., a glass). Because most common ceramics are crystalline, the definition of ceramic is often restricted to inorganic crystalline materials, as opposed to the non-crystalline glasses. The earliest ceramics were pottery objects made from clay, either by itself or mixed with other materials. This clay is often times fired in a kiln and then glazed and re-fired to create a colored, smooth surface. Ceramics now include domestic, industrial and building products and art objects etc. In the 20th century, new ceramic materials were developed for use in advanced ceramic engineering; for example, in semiconductors, superconductors etc.

Ceramic materials display a wide range of properties that facilitate their use in many different product areas. They are harder and stiffer than steel, more heat and corrosion resistant than metals or polymer's, less dense than most metals and their alloys; and their raw materials are both plentiful and inexpensive. Therefore, ceramics materials display a wide range of properties, which facilitate their use in many different product areas.

Everyday new and different applications have been discovered. Currently, there has been keen interest in the field of ceramic materials due to their excellent 
mechanical and physical properties [2]. Electronic ceramics are very useful in device fabrication. The electronic ceramics, such as, barium titanate $\left(\mathrm{BaTiO}_{3}\right)$, lead titanate $\left(\mathrm{PbTiO}_{3}\right)$, lead zirconate $\left(\mathrm{PbZrO}_{3}\right)$ with $\mathrm{ABO}_{3}$ perovskite structure show ferroelectric and anti-ferroelectric behaviors which are being used in many applications in electronics and optics. A large number of applications of these ceramic materials is the consequences of ferroelectric and anti-ferroelectric properties such as dielectric, piezoelectric, pyroelectric and electro-optic properties. Such properties can be improved and modified by chemical substitutions at $\mathrm{A}$ or $\mathrm{B}$ site of $\mathrm{ABO}_{3}$ compounds [3].

Phase transitions of these materials involve a change in atomic as well as spin or electronic configuration and can change their physical properties quite drastically [2]. More commonly, phase transition can be brought about by a change in the temperature of a system. The ferroelectric and anti-ferroelectric phase transition in perovskite has been considerable interest due to their wide range of technological applications.

Barium stannate titanate (BST) is a binary solid solution system composed of ferroelectric barium titanate and non-ferroelectric barium titanate. Both of them are of perovskite structure with $\mathrm{ABO}_{3}$. In this work, we have studied the phase transition behavior of $\left[\mathrm{Ba}_{1-\mathrm{x}} \mathrm{Sn}_{\mathrm{x}}\right] \mathrm{TiO}_{3}($ for $\mathrm{x}=0.5$ ) ceramics obtained by the dry-route method.

\section{Materials and Methods}

The perovskite samples of pure and Sn doped Barium strontium Titanate synthesized $\left(\mathrm{Ba}_{1-\mathrm{x}} \mathrm{Sn}_{\mathrm{x}}\right) \quad \mathrm{TiO}_{3}$ compositions containing $\mathrm{Ba}$ content, $\mathrm{x}=0.50$. All the samples were prepared using $\mathrm{BaCO}_{3}, \mathrm{SnCl}_{2}$ and $\mathrm{TiO}_{2}$ with assay $100 \%, 99.7 \%$ and $99 \%$ respectively. These compositions in powder form were mixed in desired molar ratios in acetone. These solid mixtures were milled for 6 hours with acetone in an indigenously designed ball-mill to produce a final powder. The powder, thus formed, was dried and then calcinated in an aluminum crucible at $1100{ }^{\circ} \mathrm{C}$ for 4 hours. The powder added with 5\% PVA (Polyvinyl alcohol) was compacted into disc-shaped pallets having diameter $1.32 \mathrm{~cm}$ using a hydraulic press at an optimized load in the range of $70 \mathrm{~N}$ to $80 \mathrm{~N}$. Then, the pellets were sintered at $1300{ }^{\circ} \mathrm{C}$ for 5 hours. The density and porosity of the pellet were measured using the sample using the liquid displacement method (glycerin displacement).
Finally, in order to measure the dielectric properties, the silver paste was painted on the polished ceramic samples as the electrodes and heated at $500{ }^{\circ} \mathrm{C}$ for 30 minutes. The dielectric properties are studied using the HIOKI 3532-50 LCR HITESTER. The instrument gives only the capacitances at a different frequency which were used to measure dielectric constant.

The synthesis of the BST ceramics and characterization following the dry-route method and outline as in Figure 1.

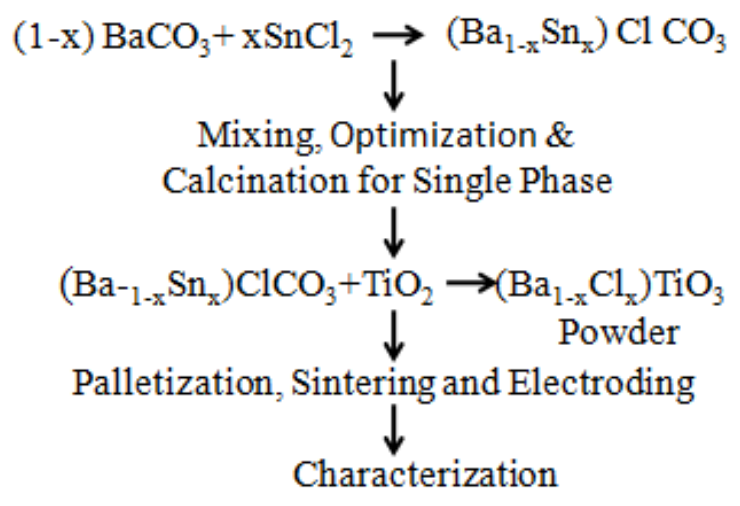

Figure 1: Details of synthesis of $\left(\mathrm{Ba}_{1-x} \mathrm{Sn}_{x}\right) \mathrm{TiO}_{3}$ ceramics by dry route method

\section{Measurement/characterization techniques}

\section{Density and porosity measurement}

The density is measured by the liquid displacement method. In the present experiment, glycerin (density $1.255 \mathrm{gm} / \mathrm{cc}$ at $20{ }^{\circ} \mathrm{C}$ ) is used as liquid and digital balance is used for taking the weight of the sample and upthrust of the liquid. The whole process depends on the Archimedes principle. The density of the sample can be calculated by using the formula,

$$
\begin{gathered}
\rho=\frac{W_{\text {air }}}{W_{\text {air }}-W_{\text {glycerine }}} \times \rho_{\text {glycerine }} \\
\text { Where } W_{\text {air }}=\text { weight of the sample in air } \\
W_{\text {glycerine }}=\text { weight of sample in glycerine } \\
\rho_{\text {glycerine }}=\text { density of glycerin at the laboratory temperature }
\end{gathered}
$$

For the measurement of porosity, we have plotted a graph between soaking time and percentage densification.

\section{Dielectric measurement}

The dielectric properties of the sample are to be studied after electroding. HIOKI 353250 LCR HITESTER is used for the study of dielectric properties 
using the dual-channel thermometer, temperature controller, sample holder and the instrument. The observed data are then used for the study in our experimental work. The instrument gives directly the capacitances at diferent frequency which are used to measure dielectric constant by the relation

$\varepsilon=\mathrm{Cd} / \varepsilon_{0} \mathrm{~A}$, where, $\varepsilon_{0}=$ dielectric constant and $\varepsilon_{r}=\varepsilon_{r}^{\prime}-i \varepsilon_{r}^{\prime \prime}$

where $\varepsilon_{r}=$ complex dielectric constant for AC filed with real part $\varepsilon_{r}^{\prime}$ and imaginary part $\varepsilon_{r}^{\prime \prime}$

$$
\varepsilon_{0}=8.854 \times 10^{-12} \mathrm{~F} / \mathrm{m}, \mathrm{d}=\text { plate separation, } \mathrm{A}=\text { area of plate }\left(=\frac{\pi D^{2}}{4}\right)
$$

where, "D" is diameter of the pellet. We have studies variation of dielectric constant i.e real and imaginary parts of various frequency with different temperatures.

\section{Results and Discussion}

\section{Density and porosity measurement}

The quality of the ceramic sample is reflected by the percentage of density and porosity. In this study, the weight of the sample in pellet form before electroding is taken by electronic balance which has the accuracy of $\pm 10^{-3} \mathrm{gm}$. Then the density of the sample is measured by liquid displacement method using glycerin of density $1.2600 \mathrm{gm} / \mathrm{cc}$ at $25^{\circ} \mathrm{C}$ and of purity of $99.5 \%$. A small amount of porosity is present in all ferroelectric ceramics and is usually considered a defect introduced due to processing imperfections; however, it may also be introduced deliberately to create a composite material system using porosity as a second component [4-6] to provide beneficial effective properties for specific applications. When such a sample is immersed into liquid, liquid

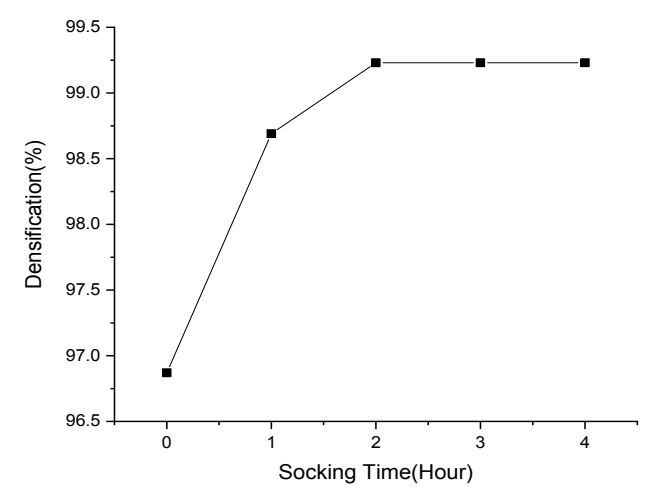

Figure 2: The variation of densification vs soaking time molecules inter into the pores resulting in the increase in mass in the given volume. From the measurement of this increased mass, the porosity of the ceramic sample was measured.

We have measured the porosity of the sample in terms of percentage of density, in another sense, it also measures the amount of water absorbed by the pores of the sample in percentage.

Figure 2 indicates that our sample is more compact due to the carefulness of processing and the increase of doping percentage.

\section{Ferroelectric to paraelectric phase transition}

Figure 3 and Figure 4 show the variation of the real and imaginary part of dielectric constant with temperature during heating of BST50 at $100 \mathrm{KHz}$

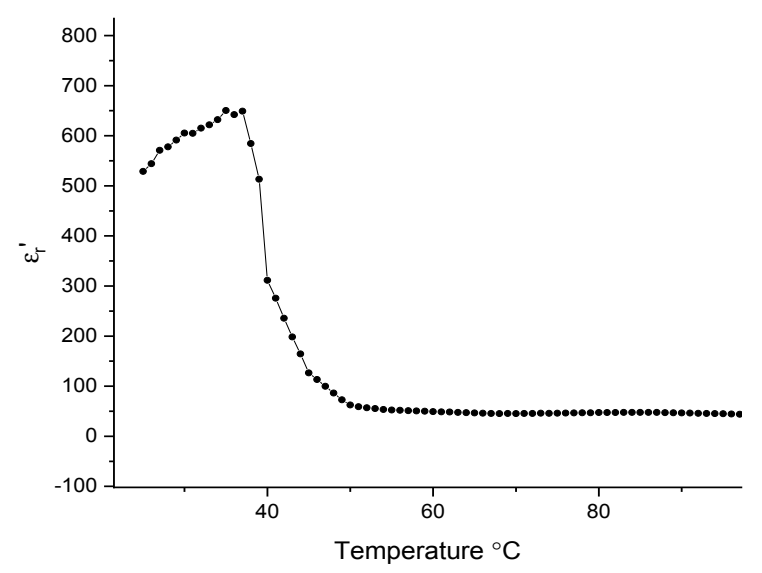

Figure 3: The variation of real part of dielectric constant with temperature for BST50 at $100 \mathrm{KHz}$.

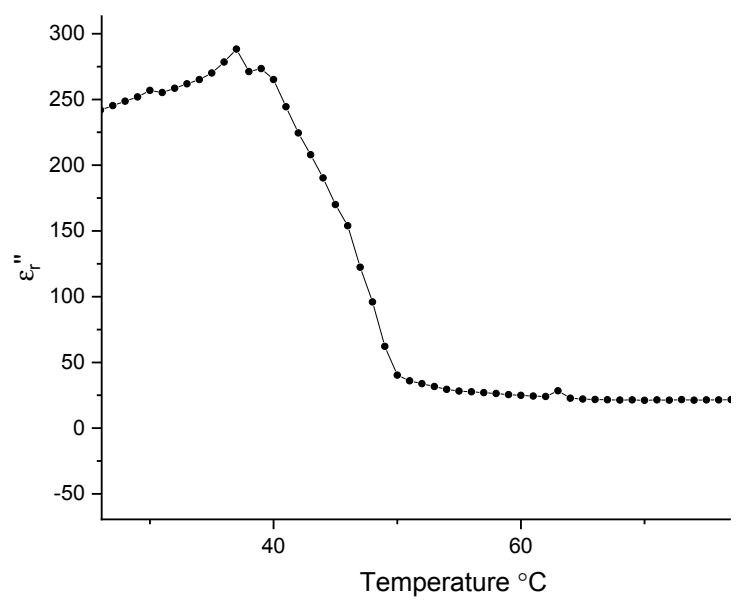

Figure 4: The variation of imaginary part of dielectric constant with temperature for BST50 at $100 \mathrm{KHz}$. 
From these figures (iii) \& (iv) it is seen that the ferroelectric to paraelectric phase transition occurs at temperatures $37.0934{ }^{\circ} \mathrm{C}$ during heating mode. The peak value of dielectric constants for heating mode is 660 whereas the corresponding value of dielectric constant at room temperature $\left(25^{\circ} \mathrm{C}\right)$ is 528.9268 [7].

\section{Curie-Weiss Law}

The dielectric constant of BST is known to exhibit Curie-Weiss behavior to temperatures very close to transition temperature [8]. The validity of CurieWeiss law for BST sample is demonstrated in Figure 5 which depict the variation of dielectric stiffness $\left(\frac{1}{\varepsilon_{r}^{\prime}}\right)$ with temperature followed by the relation;

$$
\frac{1}{\epsilon^{\prime} r}=\frac{T-T_{0}}{C}
$$

Where, $\mathrm{C}$ is Curie constant and $\mathrm{T}_{0}$ is Curie temperature. From this figure, we have calculated the Curie constant using the slope of the fitted straight line

To confirm whether the transition is diffuse or regular type, we further use Curie-Weiss like relationship:

$$
\frac{1}{\varepsilon_{r}^{\prime}}=\frac{1}{\varepsilon_{m}^{\prime}}+\frac{\left(T-T_{C}\right)^{\gamma}}{C^{\prime}}
$$

The Curie temperatures observed from the above plot for BST50 is $37{ }^{\circ} \mathrm{C}$ which is the intercept value of $\mathrm{X}$-axis in the $\varepsilon^{\prime}{ }_{r}$ versus temperature plot. The Curie temperature of our sample was less than

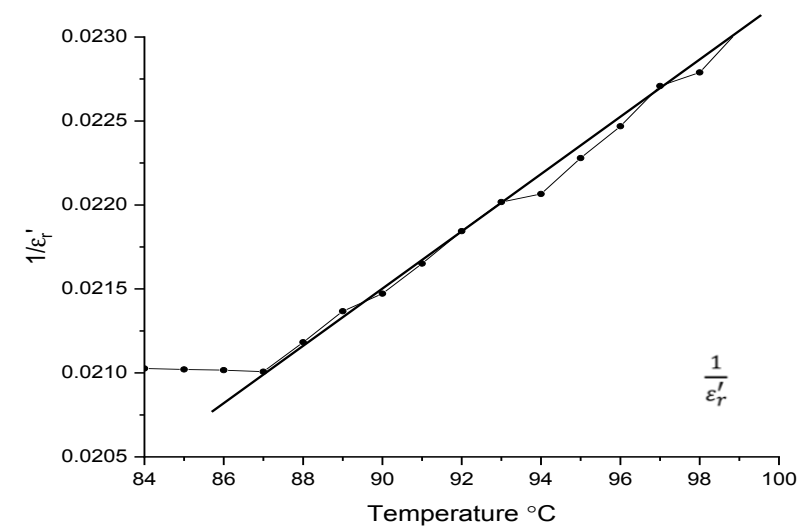

Figure 5: The ${ }_{1}$ variation of dielectric stiffness with temperature ${ }^{\overline{\varepsilon_{r}^{r}}}$ for BST50 at $100 \mathrm{KHz}$. that of transition temperature in the ferroelectric to paraelectric phase.

\section{Cole-cole study}

To check the polydisperse nature of dielectric relaxation of the dipole, a complex argand plot between $\epsilon_{r}^{\prime}$ and $\epsilon_{r}^{\prime \prime}$ called Cole-Cole plot is used [9].

The Cole-Cole plot of electrical quantities of a material is a measure of the various relaxation times for a specific dielectric material.

The complex dielectric constant in such a situation is expressed by the empirical relationship

$\varepsilon^{*}=\varepsilon^{\prime}-\varepsilon^{\prime \prime}=\varepsilon_{\infty}+\frac{\varepsilon_{s}-\varepsilon_{\infty}}{\left[1+(i \omega \tau)^{1-\alpha}\right]}$

Where, $\varepsilon_{s}$ and $\varepsilon_{\infty}$ are low and high frequency values of dielectric, $\alpha$ is a measure of the distribution; relaxation time $\tau=\frac{1}{\omega}$ and $\omega=$ $2 \pi f$

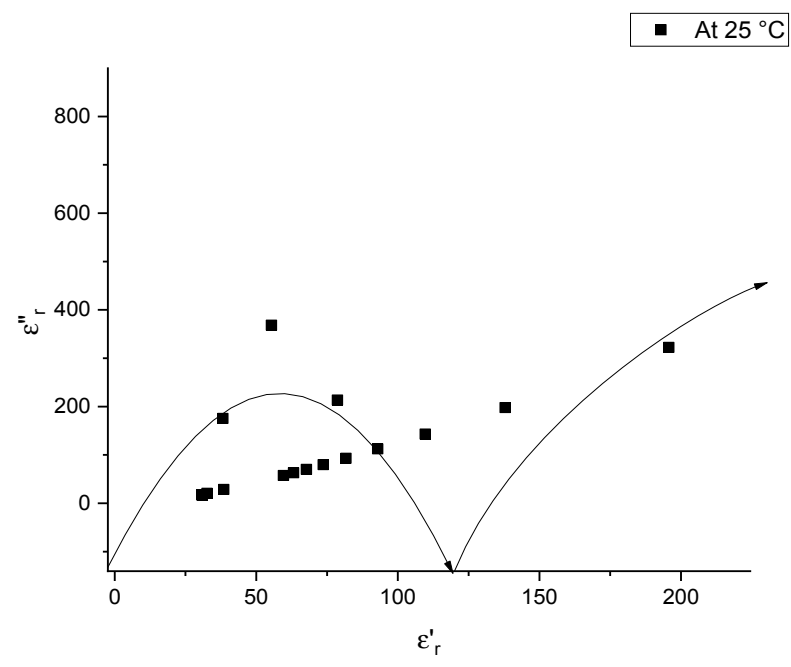

Figure 6: Cole-Cole plot between $\epsilon_{r}^{\prime}$ and $\epsilon_{r}^{\prime \prime}$ at $25^{\circ} \mathrm{C}$.

It is evident from Figure 6 that the nature of the ColeCole plot is changing with temperature. The nature of the curve is narrow semi-circular which indicates the polydisperse nature of our sample.

\section{Conclusion}

$\left(\mathrm{Ba}_{1-\mathrm{x}} \mathrm{Sn}_{\mathrm{x}}\right) \mathrm{TiO}_{3}(\mathrm{)})$ powders are prepared by a dry route method. The powders after calcination are compacted in the form of pellets using a hydraulic press at an optimized load above $70 \mathrm{KN}$. So, obtained pellets are then sintered and fired on silver paint for electroding. The sample thus formed is used for characterization. The main findings of the present work are summarized below: 
1. The experimental density of our sample measured by the liquid displacement method with glycerin is $6.1246 \mathrm{gm} / \mathrm{cc}$. whereas theoretical density is found to be $6.3224 \mathrm{gm} / \mathrm{cc}$.

2. The shape is highly dense with low porosity.

3. The structure of BST50 is tetragonal and lattice parameters of the samples $a=b=(3.86065 \pm 0.00051)$ $\AA, \mathrm{c}=(4.10234 \pm 0.00160) \AA$ and unit cell volume $(V)=(61.1440 \pm 0.0206) \AA^{3}$ with tetragonality 1.063 , which shows that on increasing the $\mathrm{Sn}^{2+}$ content volume decreases because the size of $\mathrm{Sn}^{2+}$ is smaller than that of $\mathrm{Ba}^{2+}$, in comparison to $\mathrm{BaTiO}_{3}$.

\section{Acknowledgements}

I owe a great thanks to a great many people who helped and supported me during the research of this work. I would also thank my family, friends, college faculties and Prof. Homnath Poudel (Head of Department of Physics GoldenGate International College) for their continuous support.

\section{References}

1. F. H. Riddle, Ceramic spark plug insulators, Journal of the American Ceramic Society, 1949, 32(11), 333-46. (DOI:10.1111/j.1151-2916.1949. tb18909.x).

2. A. Krell, T. Hutzler and J. Klimke, Journal of the European Ceramic Society, 2009, 29, 207-221

(DOI: 10.1016/j.jeurceramsoc. 2008.03.025)
3. T. E. Warner, Synthesis, properties and mineralogy of important inorganic materials, John Wiley \& Sons, United Kingdom, 2012, 124-144.

4. T. G. Lupeiko and S. S. Lopatin, Old and new problems in piezoelectric materials research and materials with high hydrostatic sensitivity, Inorganic Materials, 2004, 40(1), S19-S32. (DOI: 10.1023/B:INMA.0000036326.98414.3c).

5.R.Kar-Gupta andT.A. Venkatesh,Electromechanical response of porous piezoelectric materials, Acta Materialia, 2006, 54(15), 4063-4078. (DOI: 1016/j.actamat.2006.04.037)

6. R. K. Gupta and T. A. Venkatesh, Electromechanical response of piezoelectric composites: Effects of geometric connectivity and grain size, Acta Materialia, 2008, 56(15), 3810-3823. (DOI: 10.1016/j.actamat.2008.04.031)

7. B. P. Pokharel, Synthesis structure and phase transition behaviour of $\left(\mathrm{Pb}_{1-\mathrm{x}} \mathrm{Ba}_{\mathrm{x}}\right) \mathrm{ZrO}_{3}$ ceramics, $P h$. D. Thesis, Banaras Hindu University, Varanasi, India, 2000.

8. Y. Xu, Ferroelectric materials and their applications, $1^{\text {st }}$ Ed. Elsevier Science Publishing Company, London, 1991, 11-15.

9. A. Chelkowski, Dielectric Physics, Elsevier Science Publishing Company, London, 1980, 117-123. 